\title{
Infodemic: How an Epidemic of Misinformation Could Lead to a High Number of the Novel Corona Virus Disease Cases in Uganda
}

Betty K Nannyonga ${ }^{\S}$, Rhoda K Wanyeze ${ }^{\#}$, Pontiano Kaleebu ${ }^{\text {I0 }}$, John M Ssenkusu\#, Freddie Ssengooba\#, Tom Lutalo", Willford Kirungi ${ }^{\beta}$, Fredrick Edward Makumbi ${ }^{\#}$, Henry Kyobe Bosa ${ }^{q}$, Vincent A Ssembatya ${ }^{\partial}$, Henry Mwebesa ${ }^{\beta}$, Diana Atwine ${ }^{\beta}$, Jane Ruth Aceng $^{\beta}$, and Yonas Tegegn Woldermariam

${ }^{\S}$ Department of Mathematics, College of Natural Sciences, Makerere University

\#School of Public Health, College of Health Sciences, Makerere University

"Uganda Virus Research Institute

${ }^{\circ}$ MRC/UVRI \& LSHTM Uganda Research Unit

${ }^{B}$ Ministry of Health, Uganda

qUganda Peoples Defense Forces

DDirectorate of Quality Assurance Makerere University

sWorld Health Organization

\section{Abstract}

Misinformation during the COVID-19 outbreak has shaped our perception of the disease. Some people think the disease is a bioweapon while others are convinced that it is a hoax. Heightened anxiety often produces fearful rumors, some of which are absurd while others seem plausible and are laced with some truths. But, how does misinformation affect disease spread? In this paper, we construct a mathematical model parameterized by Ugandan data, to study the effect of misinformation on community COVID-19 spread. The analysis shows that misinformation leads to high number of COVID-19 cases in a community, and the effect is highest in the rumour initiators and spreaders. This analysis underscores the importance of addressing misinformation in COVID risk communication.

\section{Background}

Since December 2019, the world has grappled with an ongoing pandemic of severe acute respiratory syndrome coronavirus 2 (SARS-CoV-2) virus that causes coronavirus disease 2019 (COVID-19), with more than 5,452,503 million cases in 213 countries and territories, resulting in more than 345,068 deaths. Although $2,280,809$ million people had recovered as of May 24, 2020, the pandemic continues to cause several global socioeconomic disruptions, including the largest global recession. Misinformation or rumours about SARS-CoV-2 has spread online and has resulted in conspiracy theories regarding its origin, scale and prevention, diagnosis and treatment. With increasing concern about the COVID-19 pandemic, misinformation continues to spread from person-to-person. For example, in Nigeria, men were told to shave their beads to stay safe, and across Europe, dozens of cellphone towers were torched after the conspiracy theory linked the $5 \mathrm{G}$ technology to the pandemic. On a lighter note, misinformation was spread in India that cheering for health workers caused sound waves that weakened the virus (Neiman, 2020: Africa goes to war on COVID misinformation - with song). 
Uganda has seen her share of false information and intentional disinformation about COVID-19, with some people believing it is for the rich folks in cities. Misleading information purporting to be useful tips has gone viral on social media, including the claim that holding ones breath is a method to test for COVID-19, or that a boiled concoction of local herbs including mululuza, ettovu, niimu, ntangawuzi, mubiri cures the virus. The Madagascar coronavirus cure dubbed "Covid-Organics" (Kungu Al-Mahadi Adam, 2020: Uganda should import Madagascar's coronavirus herbal 'cure'

Uganda's status as of May 242020

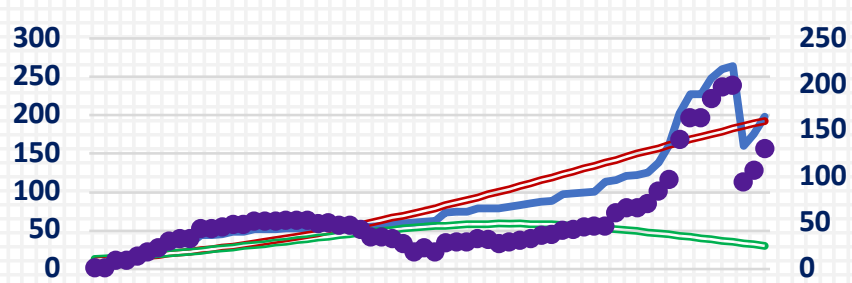

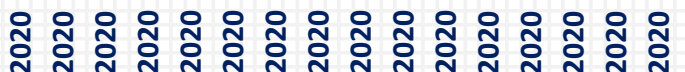
ที丶

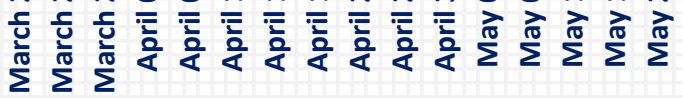

Cumulative Cases $\longrightarrow$ Logistic fit Epidemic Curve

Active Cases

Figure 1. Cumulative and active cases fit to models for testing purposes), or gargling salt or liquor, drinking hydrogen peroxide kills the virus (Shapiro, 2020: Fears of COVID-19 coronavirus provide more opportunity for misinformation about 'miracle cures') has also been acclaimed as a cure or preventive medication without any scientific trials to demonstrate its efficacy.

Misinformation is an "infodemic", and public health experts warn that it could compound the medical crisis, as social media and gossip continue to spread unfounded tips. Acting on such unofficial and unproven information can put the lives of unsuspecting public at serious risk. For this reason, the Uganda Communications Commission launched a fact-checking initiative to identify misinformation and the Ministry of Health $(\mathrm{MOH})$ has worked tirelessly to debunk false information. The World Health Organization (WHO) also declared an "infodemic" of incorrect information about the virus, which poses risks to global health.

As of May 24 2020, Uganda had predominantly reported imported cases of COVID-19, with a few sporadic local transmission cases. When almost all the initial imported cases were recovered, a sudden growth of cases was recorded due to positive truck drivers, most of whom were nationals of the neighboring east African countries. Although these imported cases were initially recorded as Ugandan cases as per WHO guidelines, following a Presidential Directive, all positive non-Ugandan truck drivers were deducted from Uganda's total confirmed case count. The status of COVID-19 in Uganda as of $24^{\text {th }}$ May 2020 is shown in Figure 1, fitted to a logistic function, and an epidemic curve.

With the increasing daily number of positive truck drivers, some Ugandans were skeptical about these cases, and did not believe that they actually existed. Others developed conspiracy theories, arguing that these were planted cases to prolong the lockdown. Many confessed their skepticism about the existence of COVID-19, which may compromise the prevention efforts. This raises many questions about the effect of misinformation on the spread of the infection after lifting of the lockdown. What would happen if such skeptical misinformed individuals contracted the deadly virus? 


\section{Research Approach}

We postulate that existence of misinformation during the COVID-19 outbreak facilitates the spread of the disease. We explore whether a contact between an ignorant and a misinformed individual is more likely to cause more corona virus infections than that between ignorant (people who are not aware of the misinformation) individuals.

Using a mathematical model, analogies between spreading of COVID-19 and dissemination of information are proposed to describe the growth and decay of the actual spreading process. Mathematical models have been developed and successfully used to study the epidemiology of diseases since the early 20th century, and of recent, a new version was developed to clarify some aspects of rumour propagation. The models have been transformed from the standard SusceptibleInfected-Removed SIR, to Ignorant-Spreader-Stifler ISS, and the mathematical techniques familiar in mathematical epidemiology applied. The model

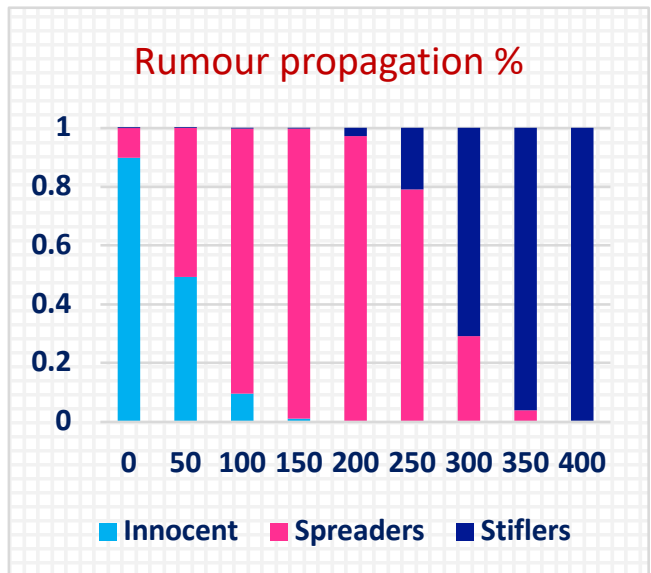

Figure 2 Rumour propagation within a community used in this analysis is a Susceptible-Infected-Removed for COVID-19 and these compartments are subdivided into Ignorant-Spreader-Stiffler depending on the rumour status of the individual. In the model, the innocent are defined as those individuals who are not misinforming people, but are at a risk of believing misinformation in circulation and may act on it. The spreaders are the misinformation initiators and propagators, while the stiflers are the informed individuals including the $\mathrm{MOH}$ and public health officials. While rumours and epidemics are popularly regarded as similar, it has long been known that there are important differences between the mathematical models for them. We show that both, along with a type of two-rumour model, can be subsumed under a general class of transient processes whose time-dependent evolution can be characterized exactly.

Results

With such assumptions, we find that rumour starts at the beginning of the epidemic, remains within the population until it becomes general knowledge (Figure 2). This implies that suppressing misinformation should mostly be done at the peak of the spread.

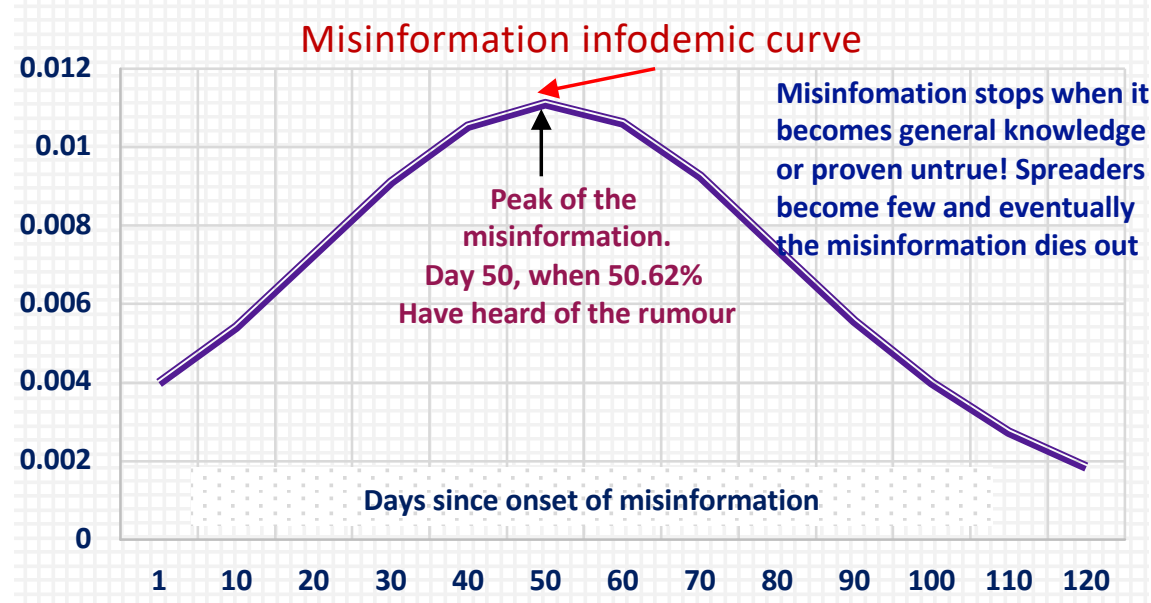

Figure 3 Rumour infodemic curve

So now we ask, when does the misinformation stop? How many individuals eventually becomes victims? Results show that it stops when it becomes general knowledge and after 
about $50.62 \%$ have heard about it (Figure 3 ), around day 50. At this point, rumour spreaders have become discouraged as an outcome of encounters either with one another or the stiflers. Like disease epidemic, we can sketch an epidemic curve for infodemic. This curve gives the peak, when optimal utilization of resources is required to stop it (Figure 4). Figure 4 shows that when more than half of the

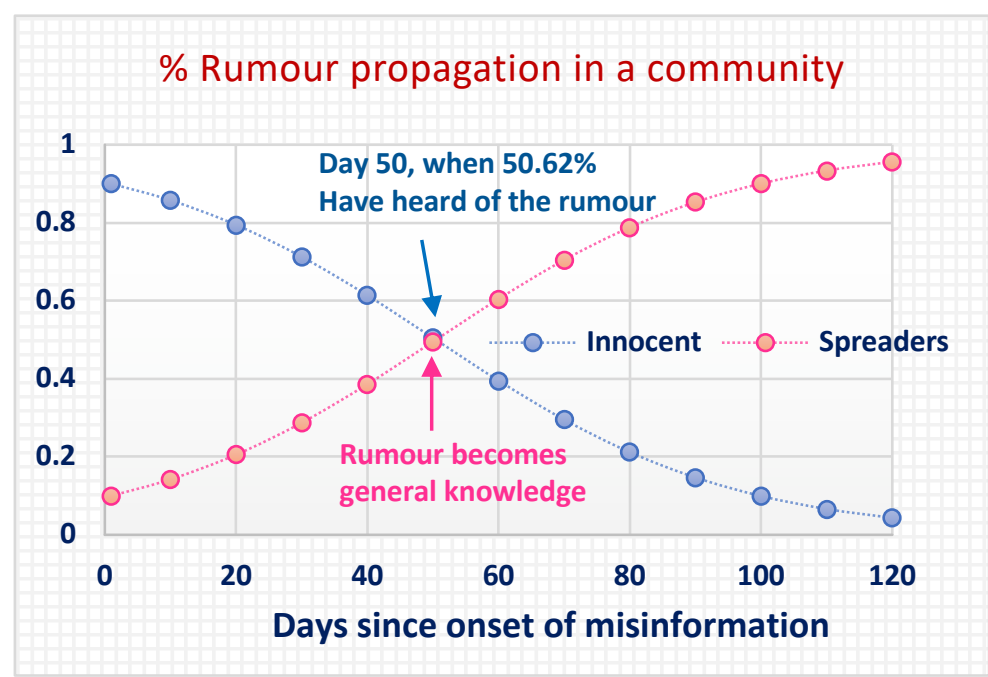
population have heard of the Figure 4 Rumour propagation rumour, it ceases to be news and the curve drops.

How many people will have been affected due to the rumour? It depends on whether they are innocent, spreaders or stiflers. Starting with only $3 \%$ of the population misinforming the public (Figure 5), it is observed that within 10 days, the rumour reaches approximately $15 \%$ of the population; i.e., 1 individual spreads to 5 new people. These 5 people spread it further to the next wave of 10, and so on (Figure 6), and the rumour continues, to 35 new people per spreader. This number slightly reduces when the disease prevails, and individuals "see" the infected person. However, there is continuity of misinformation and perhaps people switch from "preventive herbs" to what the government is (not) doing to stop or "benefit" from the epidemic. If controlled, our model shows that misinformation stops eventually, but the rate at which it does depends on the level of efficacy of the control strategy (Figure 7).

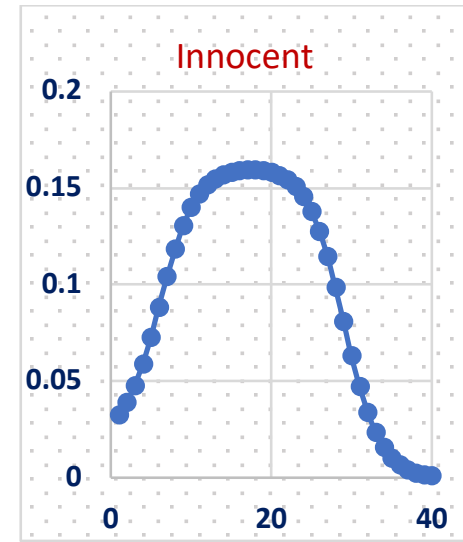

Rumour reproduction rate among the innocent. They start by believing the rumour, then eventually disregard it and recover

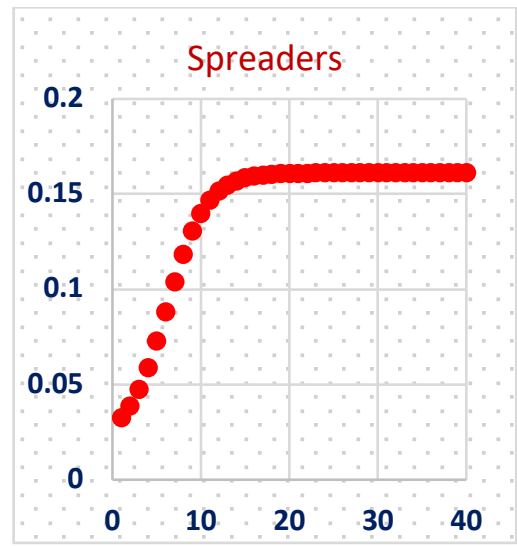

Rumour reproduction rate among the spreaders. They continue believing and spreading the rumour, until it is proven untrue or becomes general knowledge

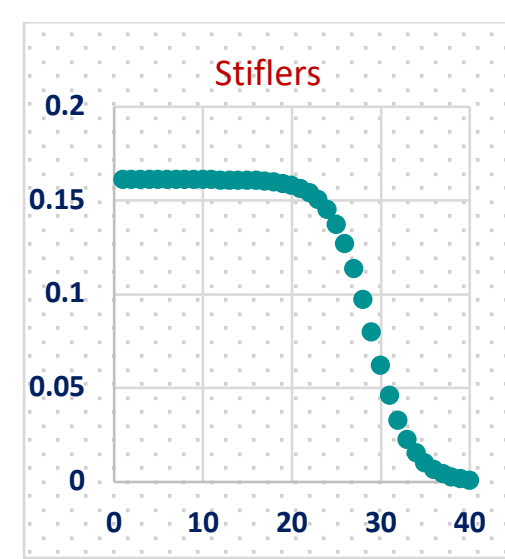

Rumour reproduction rate among the stiflers. This group includes the healthworkers and policy makers who are abreast with facts about the disease. They stop the rumour

Figure 5 Basic reproduction rate for the rumour

So how does the initial number of spreaders affect viral infection spread? This was studied starting with $100,000,200,000,300,000,400,000$ and 500,000 individuals misinforming the 
public. It was observed for example on day 17 that when rumour spreaders are many, more people get infected with the virus (see Table 1 ) and Figure 8.

We also attempt to forecast cumulative cases as a result of rumour propagation, with a hypothesis that individuals do not follow health guidelines due to this misinformation. Using nonlinear least squares method, an exponential function is obtained, that predicts the trend of cumulative cases with $95 \%$ confidence. Figure 9 gives a scenario of a possible outcome with increasing rumour spread. The fit shows that community cases can rise due to misinformation and proves a causal relation between an epidemic and infodemic.

Table 1 Number of infected individuals for each Spreader

\begin{tabular}{c|c}
\hline Initial number or Spreaders & Infected people in the community \\
\hline 100,000 & 2 \\
\hline 200,000 & 19 \\
\hline 300,000 & 79 \\
\hline 400,000 & 114 \\
\hline 500,000 & 143 \\
\hline
\end{tabular}

What can health sectors do to stop misinformation during an epidemic? Do they control the misinformation or disease? Should they control both? If so, how? What is the most effective

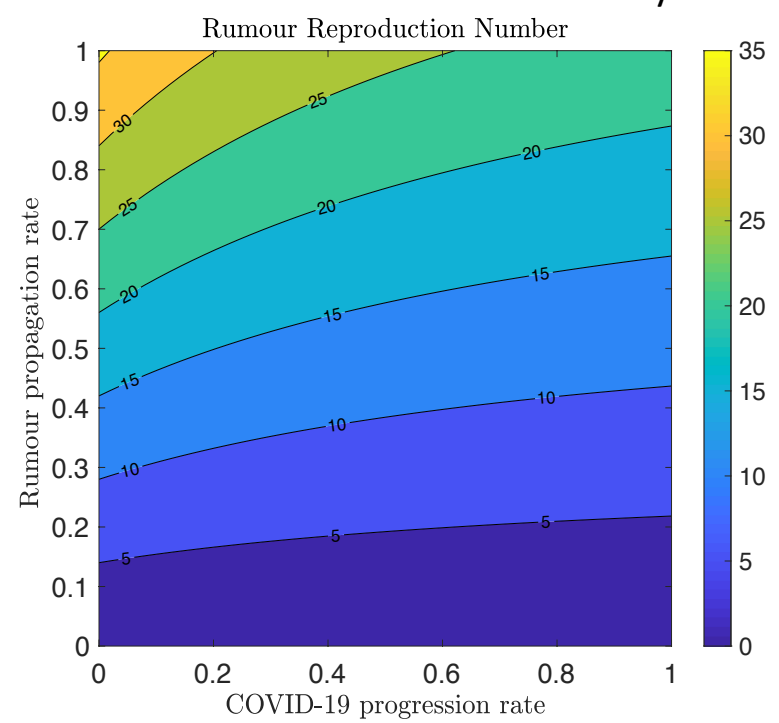

Figure 6 Rumour propagation amidst COVID-19 spread

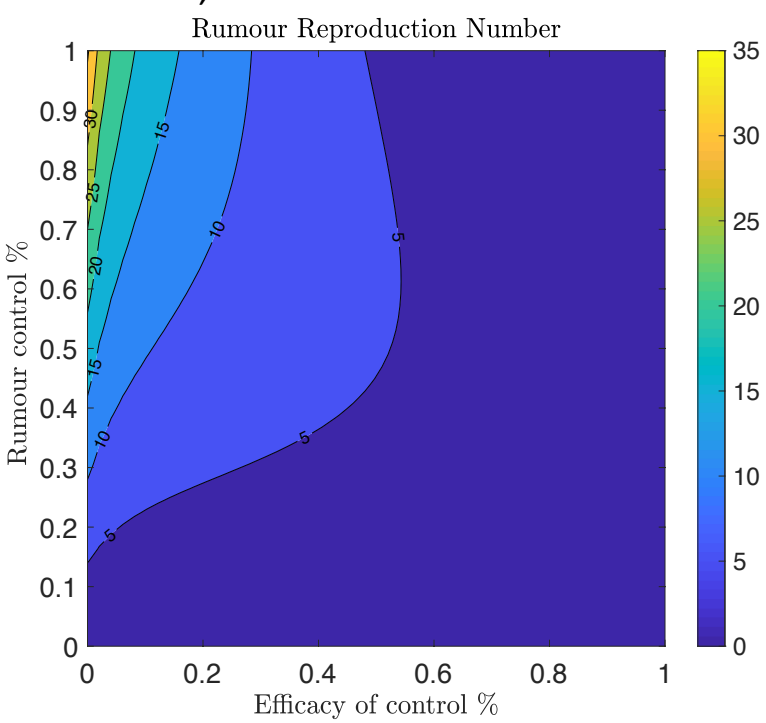

Figure 7 Rumour control and its efficacy

way of controlling rumors? Public attempts could help but it sometimes drives the spreaders underground to other forums.

\section{Discussions, Conclusions and Recommendations}

Misinformation has played an important role in the COVID-19 pandemic and spread. The information disseminated is often inaccurate, incorporating unreasoned fear and explanations of the virus's origins and questionable medical information about potential cures and treatment. The most dangerous are those where certain medical and epidemiological facts or numbers are distorted to suite one's opinion. They tend to be more believable since they are laced with known concepts, e.g. herd immunity concept and how it plays out in the COVID response. In many cases people are illiterate and more often explain the epidemic in their own terms based on misinformation. 
With the current ease and impending phased or complete lifting of the lockdown in Uganda, mitigation measures that avert community spread must be put in place. Any attempt that intercepts COVID-19 spread can save lives and socioeconomic status of the country, even when it is rumour control. This model provides a case scenario where an epidemic can be fueled by misinformation. Using Ugandan data, we estimate parameters for COVID-19 spread and fit to a model. In the case of rumour propagation we have assumed a population where 1 in 100,000 are rumour spreaders per day. We determine the rate of COVID-19 spread with increase in misinformation, the rumour production rate, the rumour infodemic curve, the effect of initial number of spreaders to disease spread and attempt to forecast cumulative cases based on percent increase in misinformation. Results for each scenario are given.

$\%$ peaks in infected spreaders with change in initial no. of spreaders

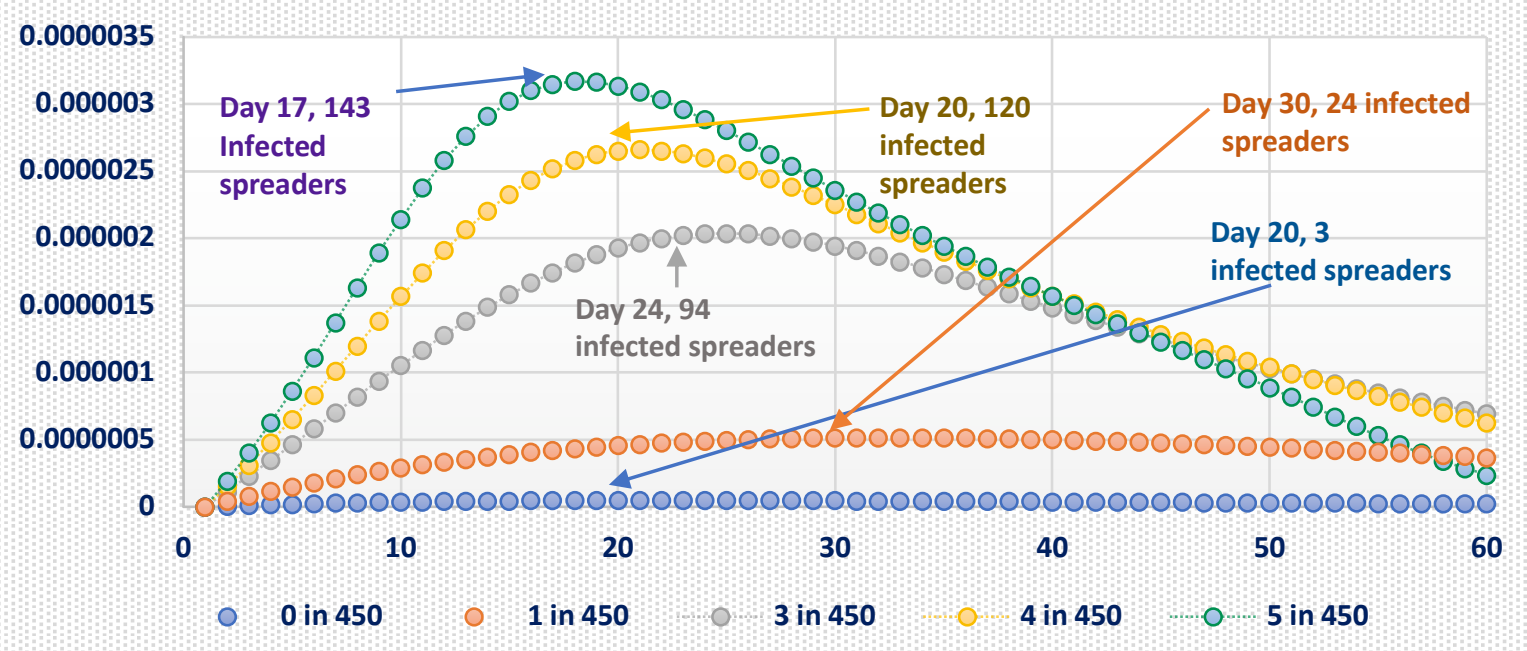

Figure 8 Number of people infected with corresponding rumour spreaders

We therefore conclude that with the impending complete lifting of the lockdown, government should consider weaving information surveillance into disease surveillance to prevent community spread in Uganda due to misinformation. Good risk communication and community mobilization might help to reduce the vulnerability of communities to search misinformation, including the frequently asked questions or addressing misinformation on concepts that frequently recur.

\section{Predicting the Trend of Cumulative Cases with Spreaders}

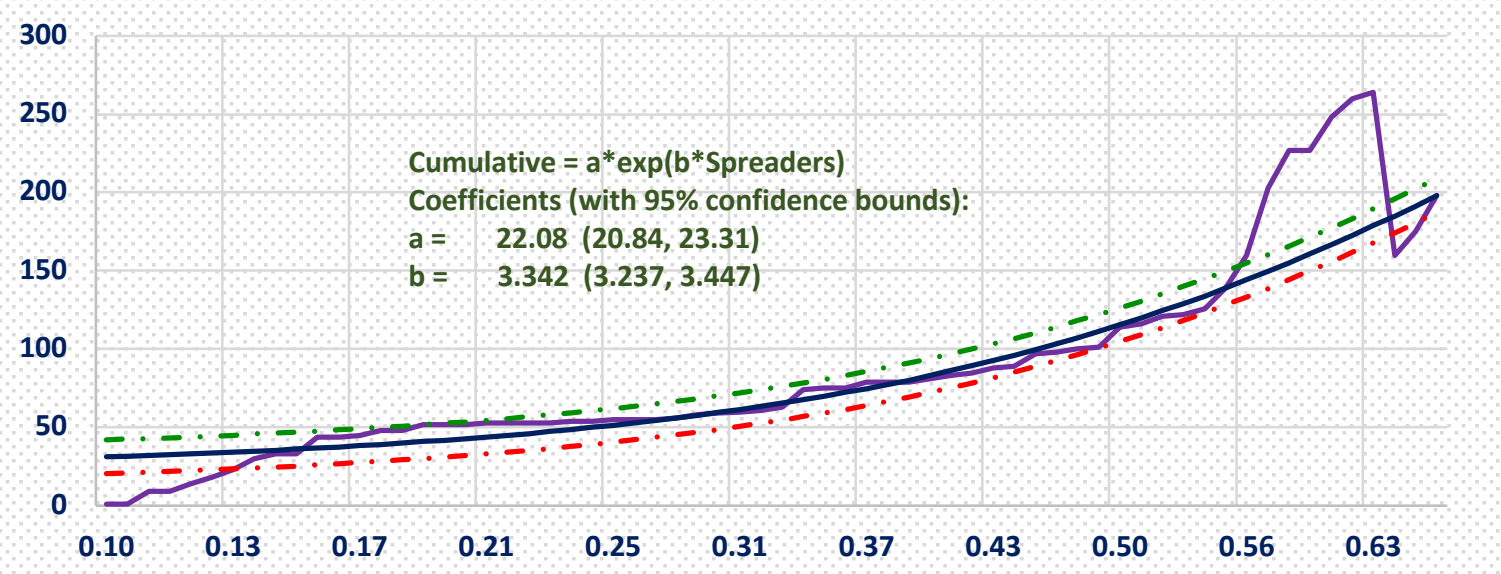

Figure 9 Forecasting Cumulative Cases based on misinformation 
We specifically propose the following strategies, among others:

1. Information surveillance should be included in disease surveillance, and techniques used to search, profile and understand rumour trends and appropriately respond to them.

2. Dissemination of the weekly COVID-19 situation reports on all media platforms to ensure that the communities are fully informed about the status of the epidemic.

3. Use of mobile Apps for information would reach further where media platforms do not.

4. Presidential and other addresses by relevant officials and experts should be conducted frequently to keep the population informed and grounded.

5. Local councils and Village Health Teams (VHTs) engage their communities about the dangers of spreading misinformation to disease outcome.

The spread of rumors in epidemics has been documented previously (Gidda, M 2016: Fear and rumours fueling the spread of Ebola). For Uganda, absence of community transmission can be and may be a huge contribution to the rumor that COVID-19 is a hoax. Timely, precise and factual information should be disseminated by the $\mathrm{MOH}$ and other respective institutions to counter such misinformation. If individuals cannot trust official sources, they cannot reject the rumor mill either (Neiman, 2020: Africa goes to war on COVID misinformation - with song). With research and collaborations between $\mathrm{MOH}, \mathrm{WHO}$, the Scientific Advisory Committee on COVID-19 in Uganda, among other stakeholders, scientists should contribute to addressing distorted science and concurrently control the infodemic and the epidemic.

\section{Acknowledgments}

The authors acknowledge all members of the Uganda COVID-19 Scientific Advisory Committee. We also acknowledge the Centers for Disease Control and Prevention partnership and contribution.

Reference

1. Sophie Neiman, Africa goes to war on COVID misinformation - with song, The Christian Science Monitor, https://www.csmonitor.com/World/Africa/2020/0428/Africa-goes-to-war-onCOVID-misinformation-with-song, Accessed May 282020.

2. Kungu Al-Mahadi Adam, Uganda should import Madagascar's coronavirus herbal 'cure' for testing purposes, SoftPower News, https://www.softpower.ug/ugandashould-import-madagascars-coronavirus-herbal-cure-for-testing-purposesmedical-experts/. Accessed May 282020.

3. Mirren Gidda, Fear and Rumors Fueling the Spread of Ebola, Time https://time.com/3092855/ebola-fear-rumors/. Accessed May 282020. 
